\title{
Variational calculation of positronium-helium-atom scattering length
}

\author{
Sadhan K. Adhikari \\ Instituto de Física Teórica, Universidade Estadual Paulista, 01.405-900 São Paulo, São Paulo, Brazil
}

(Received 8 January 2001; published 2 July 2001)

\begin{abstract}
We present a variational basis-set calculational scheme for elastic scattering of positronium atom by helium atom in $S$ wave and apply it to the calculation of the scattering length. Highly correlated trial functions with appropriate symmetry are used in this calculation. We report numerical result for the scattering length in atomic unit: $(1.0 \pm 0.1) a_{0}$. This corresponds to a zero-energy elastic cross section of (4.0 \pm 0.8$) \pi a_{0}^{2}$.

DOI: 10.1103/PhysRevA.64.022702

PACS number(s): 34.90.+q, 36.10.Dr
\end{abstract}

\section{INTRODUCTION}

Recent successful measurements of ortho positronium scattering (Ps) cross sections by $\mathrm{H}_{2}, \mathrm{~N}_{2}, \mathrm{He}, \mathrm{Ne}, \mathrm{Ar}, \mathrm{C}_{4} \mathrm{H}_{10}$, and $\mathrm{C}_{5} \mathrm{H}_{12}[1-7]$ have spurred renewed theoretical activity in this subject [8-13]. Of these, the Ps-He system is of special interest as it is the simplest system in which there are experimental results for total cross section [2-4] and pickoff quenching rate $[5,14]$. The experimental results for partial and differential cross sections for this system should be available soon [1]. A complete understanding of this system is necessary before a venture to more complex targets.

The pioneering calculations in this system using the static exchange approximation were performed by Barker and Bransden $[15,16]$ and by Fraser and Kraidy [17,18]. There have also been $R$-matrix [8], close-coupling (CC) $[11,12]$ and model-potential [19] calculations for Ps-He scattering. More recently, there has been successful calculation of Ps scattering by $\mathrm{H}$ [20], $\mathrm{He}$ [21-23], $\mathrm{Ne}$ [23], $\mathrm{Ar}$ [23], and $\mathrm{H}_{2}$ [24] using a regularized model exchange potential in a coupledchannel formulation.

However, there is considerable discrepancy among the different theoretical Ps-He cross sections at zero energy, which we discuss below. The static-exchange calculation by Sarkar and Ghosh [11], and by Blackwood et al. [8] yielded $14.38 \pi a_{0}^{2}$ (at $0.068 \mathrm{eV}$ ), and $14.58 \pi a_{0}^{2}$ (at $0 \mathrm{eV}$ ), respectively, for the elastic cross section. The inclusion of more states of Ps in the CC [12] and $R$-matrix [8] calculations does not change these results substantially. The pioneering staticexchange calculations by Barker and Bransden [15] yielded $13.04 \pi a_{0}^{2}$ and by Fraser [17] yielded $14.2 \pi a_{0}^{2}$ for zeroenergy Ps-He cross section. These results are in good agreement with each other. However, the model potential calculation by Drachman and Houston [19] yielded $7.73 \pi a_{0}^{2}$ and by this author [22] yielded 3.34 $\pi a_{0}^{2}$ for the zero-energy Ps-He cross section. So there is considerable discrepancy in the results of different theoretical calculation of low-energy Ps-He elastic scattering.

On the experimental front, there have been conflicting results for the low-energy Ps-He elastic cross section by Nagashima et al. [4], who measured a cross section of (13 土4) $\pi a_{0}^{2}$ at $0.15 \mathrm{eV}$, by Coleman et al. [6], who reported $9 \pi a_{0}^{2}$ at $0 \mathrm{eV}$, by Canter et al. [5], who found $8.47 \pi a_{0}^{2}$ at 0 $\mathrm{eV}$, and by Skalsey et al. [3], who measured (2.6 \pm 0.5$) \pi a_{0}^{2}$ at $0.9 \mathrm{eV}$. It is unlikely that these findings could be consistent with each other.

The results for the total cross section of Ps scattering obtained from the coupled-channel calculation employing the model potential [21-23] are in agreement with experiments of Refs. [1-3] at low energies. For Ps-He, this model, while it agrees [21-23] with the experimental total cross sections $[1,3]$ in the energy range 0 to $70 \mathrm{eV}$, reproduces [25] successfully the experimental pickoff quenching rate $[5,14]$. All other calculations could not reproduce the general trend of cross sections of Ps-He scattering in the energy range 0 to 70 $\mathrm{eV}$ and yielded a much too small quenching rate at thermal energies $[15,17,25]$. However, the very low-energy elastic cross sections of the model-potential calculation [21-23] are at variance with the experiments of Refs. [4-6].

Pointing at the discrepancy above among different theoretical and experimental studies, Blackwood et al. [8] called for a "fully fledged calculation" to resolve the situation. Here, we present a variational basis-set calculational scheme for low-energy Ps-He scattering in $S$ wave below the lowest Ps-excitation threshold at $5.1 \mathrm{eV}$. Using this method, we report numerical results for the scattering length of Ps-He using a one-parameter uncorrelated $\mathrm{He}$ ground-state wave function [26].

We present the formulation for the variational basis-set calculation in Sec. II, the numerical result for Ps-He scattering length in Sec. III, and a summary in Sec. IV.

\section{FORMULATION}

Because of the existence of three identical fermions (electrons) in the Ps-He system, one needs to antisymmetrize the full wave function. The position vectors of the electrons $-\mathbf{r}_{1}$ of Ps, and $\mathbf{r}_{2}$ and $\mathbf{r}_{3}$ of $\mathrm{He}-$ and positron (x) measured with respect to the massive alpha particle at the origin are shown in Fig. 1. In this configuration, the wave function for elastic scattering in the electronic doublet state of Ps-He is taken as

$$
\psi_{\mathbf{k}}^{1}(1,2,3)=\phi(2,3) \eta(1) F_{\mathbf{k}}(1,2,3) \chi(1,2,3),
$$

where $\mathbf{k}$ is the incident Ps momentum and

$$
\chi(1,2,3)=\alpha(1) \frac{1}{\sqrt{2}}[\alpha(2) \beta(3)-\beta(2) \alpha(3)],
$$




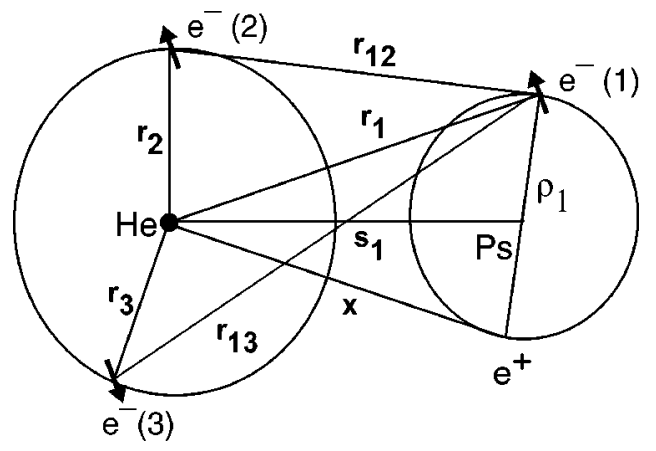

FIG. 1. Different position vectors for the Ps-He system with respect to the massive alpha particle at the origin in arrangement 1 with electrons 2 and 3 forming He and one forming Ps. The arrows on the electrons indicate the orientations of spin up and down.

represents the doublet wave function of Ps-He and where $\alpha$ denotes the spin-up state and $\beta$ denotes down and $\eta(1)$ denotes the Ps wave function of electron 1 . The He groundstate wave function $\phi(2,3)$ and the scattering function $F_{\mathbf{k}}(1,2,3)$ are symmetric under the exchange of electrons 2 and 3. The spin function $\chi(1,2,3)$ is antisymmetric under the same exchange. The full antisymmetrization operator for the three electrons is $\left(1-P_{12}-P_{13}\right)\left(1-P_{23}\right)$ where $P_{i j}$ is an operator for exchange in both space and spin of electrons $i$ and $j$. As the scattering wave function (1) above is already antisymmetrized with respect to electrons 2 and 3, the operator $\left(1-P_{23}\right)$ in the antisymmetrizer is redundant and the relevant antisymmetrizer in this case is $\mathcal{A} \equiv\left(1-P_{12}-P_{13}\right)$. Hence, the fully antisymmetric state $\psi_{\mathbf{k}}^{\mathcal{A}}$ of Ps-He scattering is given by

$$
\begin{aligned}
\psi_{\mathbf{k}}^{\mathcal{A}}= & \mathcal{A} \psi_{\mathbf{k}}^{1} \\
= & \phi(2,3) \eta(1) F_{\mathbf{k}}(1,2,3) \chi(1,2,3) \\
& -\phi(3,1) \eta(2) F_{\mathbf{k}}(2,3,1) \chi(2,3,1) \\
& -\phi(1,2) \eta(3) F_{\mathbf{k}}(3,1,2) \chi(3,1,2) .
\end{aligned}
$$

The projection of the Schrödinger equation $(H-E)\left|\psi_{\mathbf{k}}^{\mathcal{A}}\right\rangle$ $=0$ on the doublet state $\chi(1,2,3)$ is

$$
\begin{aligned}
\left\langle\chi(1,2,3)|(H-E)| \psi_{\mathbf{k}}^{\mathcal{A}}\right\rangle= & (H-E) \mid \phi(2,3) \eta(1) F_{\mathbf{k}}(1,2,3) \\
& \left.-\phi(3,1) \eta(2) F_{\mathbf{k}}(2,3,1)\right\rangle=0,
\end{aligned}
$$

with $H$ the full Ps-He Hamiltonian. The incident Ps energy $E=6.8 k^{2} \mathrm{eV}$. Using the identities $\langle\chi(1,2,3) \mid \chi(1,2,3)\rangle=1$ and $\langle\chi(1,2,3) \mid \chi(2,3,1)\rangle=\langle\chi(1,2,3) \mid \chi(3,1,2)\rangle=1 / 2$, we see that the last two terms on the right-hand side of Eq. (3) give equivalent contribution that are combined in Eq. (4), which is rewritten as

$$
(H-E)\left(1-P_{12}\right)\left|\phi(2,3) \eta(1) F_{\mathbf{k}}(1,2,3)\right\rangle=0 .
$$

Hence, after the spin projection to the doublet state the effective antisymmetrizer to be used on state (1) is $\mathcal{A}_{1} \equiv(1$
$\left.-P_{12}\right)$. We shall use this antisymmetrizer in the following and suppress the spin functions.

The full Ps-He Hamiltonian $H$ can be broken in the convenient form as follows: $H=H_{1}+V_{1}$ where $H_{1}$ includes the full kinetic energy and intracluster interaction of $\mathrm{He}$ and Ps for the arrangement shown in Fig. 1 and $V_{1}$ is the sum of the intercluster interaction between $\mathrm{He}$ and $\mathrm{Ps}$ in the same configuration:

$$
V_{1}=\left[\frac{2}{x}-\frac{2}{r_{1}}+\frac{1}{r_{12}}-\frac{1}{\rho_{2}}+\frac{1}{r_{13}}-\frac{1}{\rho_{3}}\right] .
$$

We employ the position vectors $\mathbf{s}_{j}=\left(\mathbf{x}+\mathbf{r}_{j}\right) / 2, \rho_{j}=\mathbf{x}-\mathbf{r}_{j}$, $\mathbf{r}_{i j}=\mathbf{r}_{i}-\mathbf{r}_{j}, i, j=1,2,3, i \neq j$.

The fully antisymmetric state satisfies the LippmannSchwinger equation [27]

$$
\begin{gathered}
\left|\psi_{\mathbf{k}}^{1}\right\rangle=\left|\phi_{\mathbf{k}}^{1}\right\rangle+G_{1} M_{1}\left|\psi_{\mathbf{k}}^{1}\right\rangle, \\
M_{1}=V_{1} \mathcal{A}_{1}+\left(E-H_{1}\right)\left(1-\mathcal{A}_{1}\right) \equiv \mathcal{A}_{1} V_{1}+\left(1-\mathcal{A}_{1}\right)\left(E-H_{1}\right),
\end{gathered}
$$

where the channel Green's function is given by $G_{1} \equiv(E$ $\left.+i 0-H_{1}\right)^{-1}$ and the incident wave $\left|\phi_{\mathbf{k}}^{1}\right\rangle$ satisfies ( $E$ $\left.-H_{1}\right)\left|\phi_{\mathbf{k}}^{1}\right\rangle=0$. We are using atomic units (au) in which $a_{0}$ $=e=m=\hbar=1$, where $e(m)$ is the electronic charge (mass) and $a_{0}$ the Bohr radius.

The properly symmetrized transition matrix for elastic scattering is defined by $\left\langle\phi_{\mathbf{k}}^{1}\left|T^{\mathcal{A}}\right| \phi_{\mathbf{k}}^{1}\right\rangle=\left\langle\phi_{\mathbf{k}}^{1}\left|V_{1}\right| \psi_{\mathbf{k}}^{\mathcal{A}}\right\rangle$ $=\left\langle\phi_{\mathbf{k}}^{1}\left|V_{1} \mathcal{A}_{1}\right| \psi_{\mathbf{k}}^{1}\right\rangle=\left\langle\psi_{\mathbf{k}}^{1}\left|\mathcal{A}_{1} V_{1}\right| \phi_{\mathbf{k}}^{1}\right\rangle$ [27]. A basis-set calculational scheme for the transition matrix can be obtained from the following expression [28]

$$
\begin{aligned}
\left\langle\phi_{\mathbf{k}}^{1}\left|T^{\mathcal{A}}\right| \phi_{\mathbf{k}}^{1}\right\rangle= & \left\langle\psi_{\mathbf{k}}^{1}\left|\mathcal{A}_{1} V_{1}\right| \phi_{\mathbf{k}}^{1}\right\rangle+\left\langle\phi_{\mathbf{k}}^{1}\left|\mathcal{A}_{1} V_{1}\right| \psi_{\mathbf{k}}^{1}\right\rangle \\
& -\left\langle\psi_{\mathbf{k}}^{1}\left|\mathcal{A}_{1} V_{1}-M_{1} G_{1} \mathcal{A}_{1} V_{1}\right| \psi_{\mathbf{k}}^{1}\right\rangle .
\end{aligned}
$$

Using Eq. (7), it can be verified that Eq. (9) is an identity if exact-scattering wave functions $\psi_{\mathbf{k}}^{1}$ are used. If approximate wave functions are used, expression (9) is stationary with respect to small variations of $\left|\psi_{\mathbf{k}}^{1}\right\rangle$ but not of $\left\langle\psi_{\mathbf{k}}^{1}\right|$. This onesided variational property emerges because of the lack of symmetry of the formulation in the presence of explicit antisymmetrization operator $\mathcal{A}_{1}$. However, this variational property can be used to formulate a basis-set calculational scheme with the following trial functions [28]

$$
\left|\psi_{\mathbf{k}}^{1}\right\rangle_{t}=\sum_{n=1}^{N} a_{n}\left|f_{n}\right\rangle, \quad{ }_{t}\left\langle\psi_{\mathbf{k}}^{1}\right|=\sum_{m=1}^{N} b_{m}\left\langle f_{m}\right|
$$

where the suffix $t$ denotes trial and $f_{n}, n=1,2, \ldots, N$, are the basis functions. Substituting Eq. (10) into Eq. (9) and using this variational property with respect to $\left|\psi_{\mathbf{k}}^{1}\right\rangle$ we obtain [28]

$$
\begin{aligned}
{ }_{t}\left\langle\psi_{\mathbf{k}}^{1}\right|=\sum_{m=1}^{N}\left\langle\phi_{\mathbf{k}}^{1}\left|\mathcal{A}_{1} V_{1}\right| f_{n}\right\rangle D_{n m}\left\langle f_{m}\right|, \\
\left(D^{-1}\right)_{m n}=\left\langle f_{m}\right| \mathcal{A}_{1} V_{1}-\left[\mathcal{A}_{1} V_{1}+\left(1-\mathcal{A}_{1}\right)\left(E-H_{1}\right)\right] \\
\times G_{1} \mathcal{A}_{1} V_{1}\left|f_{n}\right\rangle .
\end{aligned}
$$


Using the variational form (11) and definition $\left\langle\phi_{\mathbf{k}}^{1}\left|T^{\mathcal{A}}\right| \phi_{\mathbf{k}}^{1}\right\rangle$ $=\left\langle\psi_{\mathbf{k}}^{1}\left|\mathcal{A}_{1} V_{1}\right| \phi_{\mathbf{k}}^{1}\right\rangle$ we obtain the following basis-set calculational scheme for the transition matrix

$$
\left\langle\phi_{\mathbf{k}}^{1}\left|T^{\mathcal{A}}\right| \phi_{\mathbf{k}}^{1}\right\rangle_{t}=\sum_{m, n=1}^{N}\left\langle\phi_{\mathbf{k}}^{1}\left|\mathcal{A}_{1} V_{1}\right| f_{n}\right\rangle D_{n m}\left\langle f_{m}\left|\mathcal{A}_{1} V_{1}\right| \phi_{\mathbf{k}}^{1}\right\rangle
$$

Equations (12) and (13) are also valid in partial-wave form.

In the present $S$-wave calculation, the basis functions are taken in the following form

$$
\begin{aligned}
& f_{m}\left(\mathbf{r}_{2}, \mathbf{r}_{3}, \rho_{1}, \mathbf{s}_{1}\right)=\varphi\left(\mathbf{r}_{3}\right) g_{m}\left(\mathbf{r}_{2}, \rho_{1}, \mathbf{s}_{1}\right) \\
& g_{m}\left(\mathbf{r}_{2}, \rho_{1}, \mathbf{s}_{1}\right)= \varphi\left(\mathbf{r}_{2}\right) \eta\left(\rho_{1}\right) \\
& \times e^{-\delta_{m} r_{2}-\alpha_{m} \rho_{1}-\beta_{m} s_{1}-\gamma_{m}\left(\rho_{2}+r_{12}\right)-\mu_{m}\left(x+r_{1}\right)} \\
& \times \frac{\sin \left(k s_{1}\right)}{k s_{1}}
\end{aligned}
$$

where $\delta_{m}, \alpha_{m}, \beta_{m}, \gamma_{m}$, and $\mu_{m}$ are nonlinear variational parameters. The ground-state wave function of the $\mathrm{He}$ atom is taken to be $\phi\left(\mathbf{r}_{2}, \mathbf{r}_{3}\right)=\varphi\left(\mathbf{r}_{2}\right) \varphi\left(\mathbf{r}_{3}\right)$ with $\varphi(\mathbf{r})=\lambda^{3 / 2} \exp$ $(-\lambda r) / \sqrt{\pi}$ and $\lambda=1.6875 \quad[26]$ and $\eta(\rho)=\exp$ $(-0.5 \rho) / \sqrt{8 \pi}$ represent the $\operatorname{Ps}(1 s)$ wave function. For elastic scattering, the direct Born amplitude is zero and the exchange correlation dominates scattering. To be consistent with this, the direct terms in the form factors $\left\langle f_{m}\left|\mathcal{A}_{1} V_{1}\right| \phi_{\mathbf{k}}^{1}\right\rangle$ and $\left\langle\phi_{\mathbf{k}}^{1}\left|\mathcal{A}_{1} V_{1}\right| f_{n}\right\rangle$ are zero with the above choice of correlations in the basis functions via $\gamma_{m}$ and $\mu_{m}$. This property follows as the above function is invariant with respect to the interchange of $\mathbf{x}$ and $\mathbf{r}_{1}$, whereas the remaining part of the integrand in the direct terms changes sign under this transformation. In Ps-He elastic scattering, the electron 2 of $\mathrm{He}$ is the active electron undergoing exchange with the electron 1 of Ps, whereas the electron 3 of $\mathrm{He}$ is a passive spectator. In this calculation, we include in Eq. (15) correlation between electrons 1 and 2. Consequently, we deal with integrals in three vector variables $-\mathbf{r}_{1}, \mathbf{r}_{2}$, and $\mathbf{x}$. If we also include correlation involving electron 3 we shall have to deal with integration in four-vector variables, which is beyond the scope of the present study. However, we believe that a meaningful calculation can be performed only with correlation between the active electrons 1 and 2. Hence, to avoid complication, we ignore correlation involving electron 3 , which is expected to lead to correction over the present study.

In the $S$ wave at zero energy, $\sin \left(k s_{1}\right) /\left(k s_{1}\right)=1$ in Eq. (15); also, $\left|\phi_{p}^{1}\right\rangle=\varphi\left(\mathbf{r}_{2}\right) \varphi\left(\mathbf{r}_{3}\right) \eta\left(\rho_{1}\right) \sin \left(p s_{1}\right) /\left(p s_{1}\right)$. The useful matrix elements of the present approach are explicitly written as [28]

$$
\begin{aligned}
\left\langle\phi_{p}^{1}\left|\mathcal{A}_{1} V_{1}\right| f_{n}\right\rangle= & -\frac{1}{2 \pi} \int \varphi\left(\mathbf{r}_{1}\right) \varphi\left(\mathbf{r}_{3}\right) \eta\left(\rho_{2}\right) \frac{\sin \left(p s_{2}\right)}{p s_{2}}\left[V_{1}\right] \\
& \times f_{n}\left(\mathbf{r}_{2}, \mathbf{r}_{3}, \rho_{1}, \mathbf{s}_{1}\right) d \mathbf{r}_{2} d \mathbf{r}_{3} d \rho_{1} d \mathbf{s}_{1}
\end{aligned}
$$

$$
\begin{aligned}
= & -\frac{1}{2 \pi} \int \varphi\left(\mathbf{r}_{1}\right) \eta\left(\rho_{2}\right) \frac{\sin \left(p s_{2}\right)}{p s_{2}}\left[\mathcal{V}_{1}\right] \\
& \times g_{n}\left(\mathbf{r}_{2}, \rho_{1}, \mathbf{s}_{1}\right) d \mathbf{r}_{2} d \rho_{1} d \mathbf{s}_{1}, \\
\left\langle f_{m}\left|\mathcal{A}_{1} V_{1}\right| \phi_{p}^{1}\right\rangle= & -\frac{1}{2 \pi} \int g_{m}\left(\mathbf{r}_{1}, \rho_{2}, \mathbf{s}_{2}\right)\left[\mathcal{V}_{1}\right] \\
& \times \varphi\left(\mathbf{r}_{2}\right) \eta\left(\rho_{1}\right) \frac{\sin \left(p s_{1}\right)}{p s_{1}} d \mathbf{r}_{2} d \rho_{1} d \mathbf{s}_{1},
\end{aligned}
$$

$$
\begin{aligned}
\left\langle f_{m}\left|\mathcal{A}_{1} V_{1}\right| f_{n}\right\rangle= & -\frac{1}{4 \pi} \int g_{m}\left(\mathbf{r}_{1}, \rho_{2}, \mathbf{s}_{2}\right)\left[\mathcal{V}_{1}\right] \\
& \times g_{n}\left(\mathbf{r}_{2}, \rho_{1}, \mathbf{s}_{1}\right) d \mathbf{r}_{2} d \rho_{1} d \mathbf{s}_{1},
\end{aligned}
$$

with

$$
\begin{gathered}
\mathcal{V}_{1}=\left[h(x)-h\left(r_{1}\right)+\frac{1}{r_{12}}-\frac{1}{\rho_{2}}\right], \\
h(x)=\frac{1}{x}+\frac{\exp (-2 \lambda x)}{x}+\lambda \exp (-2 \lambda x), \\
\left\langle f_{m}\left|M_{1} G_{1} \mathcal{A}_{1} V_{1}\right| f_{n}\right\rangle \approx-\frac{2}{\pi} \int_{0}^{\infty} d p\left\langle f_{m}\left|\mathcal{A}_{1} V_{1}\right| \phi_{p}^{1}\right\rangle \\
\times\left\langle\phi_{p}^{1}\left|\mathcal{A}_{1} V_{1}\right| f_{n}\right\rangle,
\end{gathered}
$$

where the so-called off-shell term $\left(1-\mathcal{A}_{1}\right)\left(E-H_{1}\right)$ has been neglected for numerical simplification in this calculation. This term is expected to contribute to refinement over the present calculation. In this convention, the on-shell $t$-matrix element at zero energy is the scattering length: $a$ $=\left\langle\phi_{0}^{1}\left|T^{\mathcal{A}}\right| \phi_{0}^{1}\right\rangle$.

All the matrix elements above can be evaluated by a method presented in Refs. [29]. We describe it in the following for $\left\langle\phi_{p}^{1}\left|\mathcal{A}_{1} V_{1}\right| f_{n}\right\rangle$ of Eq. (16). By a transformation of variables from $\left(\mathbf{r}_{2}, \rho_{1}, \mathbf{s}_{1}\right)$ to $\left(\mathbf{s}_{1}, \mathbf{s}_{2}, \mathbf{x}\right)$ with Jacobian $2^{6}$ and separating the radial and angular integrations, the form factor (16) is given by

$$
\begin{aligned}
\left\langle\phi_{p}^{1}\left|\mathcal{A}_{1} V_{1}\right| f_{n}\right\rangle= & -\frac{2^{6} \lambda^{3}}{16 \pi^{3}} \int_{0}^{\infty} d s_{2} s_{2}^{2} \frac{\sin \left(p s_{2}\right)}{p s_{2}} \int_{0}^{\infty} d s_{1} s_{1}^{2} \\
& \times e^{-\beta_{n} s_{1}} \int_{0}^{\infty} d x x^{2} e^{-\mu_{n} x} \int e^{-\left(a r_{1}+b \rho_{1} / 2\right)} \\
& \times e^{-\left(c r_{2}+d \rho_{2} / 2\right)} e^{-\gamma_{n} r_{12}}\left[V_{1}\right] d \hat{s}_{1} d \hat{s}_{2} d \hat{x}
\end{aligned}
$$

where $a=\lambda+\mu_{n}, b=2 \alpha_{n}+1, c=\lambda+\delta_{n}$, and $d=2 \gamma_{n}+1$. Recalling that $\mathbf{r}_{j}=2 \mathbf{s}_{j}-\mathbf{x}, \quad \mathbf{r}_{12}=2\left(\mathbf{s}_{1}-\mathbf{s}_{2}\right), \rho_{j}=2\left(\mathbf{x}-\mathbf{s}_{j}\right), j$ $=1,2$, we employ the following expansions of the exponentials in Eq. (22) 


$$
\begin{array}{r}
e^{-a|2 \mathbf{s}-\mathbf{x}|-b|\mathbf{x}-\mathbf{s}|}=\frac{4 \pi}{s x} \sum_{l m} G_{l}^{(a, b)}(s, x) Y_{l m}^{*}(\hat{s}) Y_{l m}(\hat{x}), \\
h(|2 \mathbf{s}-\mathbf{x}|) e^{-a|2 \mathbf{s}-\mathbf{x}|-b|\mathbf{x}-\mathbf{s}|} \\
=\frac{4 \pi}{s x} \sum_{l m} J_{l}^{(a, b)}(s, x) Y_{l m}^{*}(\hat{s}) Y_{l m}(\hat{x}), \\
\frac{e^{-a|2 \mathbf{s}-\mathbf{x}|-b|\mathbf{x}-\mathbf{s}|}}{|\mathbf{s}-\mathbf{x}|}=\frac{4 \pi}{s x} \sum_{l m} K_{l}^{(a, b)}(s, x) Y_{l m}^{*}(\hat{s}) Y_{l m}(\hat{x}),
\end{array}
$$

$$
e^{-a\left|\mathbf{s}_{1}-\mathbf{s}_{2}\right|}=\frac{4 \pi}{s_{1} s_{2}} \sum_{l m} B_{l}^{(a)}\left(s_{1}, s_{2}\right) Y_{l m}^{*}\left(\hat{s}_{1}\right) Y_{l m}\left(\hat{s}_{2}\right),
$$

where the $Y_{l m}$ 's are the usual spherical harmonics. Using

$$
\begin{aligned}
\left\langle\phi_{p}^{1}\left|\mathcal{A}_{1} V_{1}\right| f_{n}\right\rangle= & -2^{8} \lambda^{3} \int_{0}^{\infty} d s_{1} e^{-\beta_{n} s_{1}} \int_{0}^{\infty} d s_{2} \frac{\sin \left(p s_{2}\right)}{p s_{2}} \int_{0}^{\infty} d x e^{-\mu_{n} x} \sum_{l=0}^{L}(2 l+1)\left[h(x) G_{l}^{(a, b)}\left(s_{1}, x\right) G_{l}^{(c, d)}\left(s_{2}, x\right) B_{l}^{\left(2 \gamma_{n}\right)}\left(s_{1}, s_{2}\right)\right. \\
& -J_{l}^{(a, b)}\left(s_{1}, x\right) G_{l}^{(c, d)}\left(s_{2}, x\right) B_{l}^{\left(2 \gamma_{n}\right)}\left(s_{1}, s_{2}\right)+\frac{1}{2} G_{l}^{(a, b)}\left(s_{1}, x\right) G_{l}^{(c, d)}\left(s_{2}, x\right) A_{l}^{\left(2 \gamma_{n}\right)}\left(s_{1}, s_{2}\right) \\
& \left.-\frac{1}{2} G_{l}^{(a, b)}\left(s_{1}, x\right) K_{l}^{(c, d)}\left(s_{2}, x\right) B_{l}^{\left(2 \gamma_{n}\right)}\left(s_{1}, s_{2}\right)\right]
\end{aligned}
$$

where the $l$ sum is truncated at $l=L$. This procedure avoids complicated angular integrations involving $\mathbf{s}_{1}, \mathbf{s}_{2}$, and $\mathbf{x}$. The matrix element takes a simple form requiring straightforward numerical computation of certain radial integrals only. The functions $G_{l}, J_{l}, K_{l}$, etc., are easily calculated using Eqs. (23)-(27):

$$
G_{l}^{(a, b)}(s, x)=\frac{s x}{2} \int_{-1}^{1} d u P_{l}(u) e^{-a|2 \mathbf{s}-\mathbf{x}|-b|\mathbf{x}-\mathbf{s}|},
$$

where $P_{l}(u)$ is the usual Legendre polynomial and $u$ is the cosine of the angle between $\mathbf{s}$ and $\mathbf{x}$. The integrals (18) and (19) can be evaluated similarly. For example,

$$
\begin{aligned}
\left\langle f_{m}\left|\mathcal{A}_{1} V_{1}\right| f_{n}\right\rangle= & -2^{7} \lambda^{3} \int_{0}^{\infty} d s_{1} e^{-\beta_{n} s_{1}} \int_{0}^{\infty} d s_{2} e^{-\beta_{m} s_{2}} \int_{0}^{\infty} d x e^{-\left(\mu_{n}+\mu_{m}\right) x} \sum_{l=0}^{L}(2 l+1) \\
& \times\left[h(x) G_{l}^{(e, f)}\left(s_{1}, x\right) G_{l}^{(g, h)}\left(s_{2}, x\right) B_{l}^{\left(2 \gamma_{m n}\right)}\left(s_{1}, s_{2}\right)-J_{l}^{(e, f)}\left(s_{1}, x\right) G_{l}^{(g, h)}\left(s_{2}, x\right) B_{l}^{\left(2 \gamma_{m n}\right)}\left(s_{1}, s_{2}\right)\right. \\
& \left.+\frac{1}{2} G_{l}^{(e, f)}\left(s_{1}, x\right) G_{l}^{(g, h)}\left(s_{2}, x\right) A_{l}^{\left(2 \gamma_{m n}\right)}\left(s_{1}, s_{2}\right)-\frac{1}{2} G_{l}^{(e, f)}\left(s_{1}, x\right) K_{l}^{(g, h)}\left(s_{2}, x\right) B_{l}^{\left(2 \gamma_{m n}\right)}\left(s_{1}, s_{2}\right)\right],
\end{aligned}
$$

where $e=\lambda+\delta_{m}+\mu_{n}, f=2 \alpha_{n}+2 \gamma_{m}+1, g=\lambda+\delta_{n}+\mu_{m}$, $h=2 \alpha_{m}+2 \gamma_{n}+1$, and $\gamma_{m n}=\gamma_{m}+\gamma_{n}$.

\section{NUMERICAL RESULT}

We tested the convergence of the integrals by varying the number of integration points in the $x, s_{1}$, and $s_{2}$ integrals in Eqs. (28) and (30) and the $u$ integral in Eq. (29). The $x$ integration was relatively easy and 20 Gauss-Legendre quadrature points appropriately distributed between 0 and 16 were enough for convergence. In the evaluation of integrals of type (29), 40 Gauss-Legendre quadrature points were suf- ficient for adequate convergence. The convergence in the numerical integration over $s_{1}$ and $s_{2}$ was achieved with 300 Gauss-Legendre quadrature points between 0 and 12 . The maximum value of $l$ in the sum in Eqs. (28) and (30), L, is taken to be 7 , which is sufficient for obtaining the convergence with the partial-wave expansions (23)-(27).

We find that a judicial choice of the parameters in Eq. (15) is needed for convergence. The present method does not provide a bound on the result. Consequently, the method could lead to a wrong scattering length if an inappropriate (incomplete) basis set is chosen. After some experimentation, we find that for good convergence, the nonlinear parameters $\delta_{n}$ and $\alpha_{n}$ should be taken to have both positive and negative 
TABLE I. Ps-He scattering length in (a.u.) for different $L$ and $N$.

\begin{tabular}{rrrrrrrr}
\hline \hline$N$ & \multicolumn{1}{c}{$L=0$} & $L=2$ & $L=3$ & $L=4$ & $L=5$ & $L=6$ & $L=7$ \\
\hline 1 & 2.056 & 1.867 & 1.782 & 1.721 & 1.681 & 1.655 & 1.638 \\
3 & 2.418 & -1.662 & 28.016 & 4.151 & 3.061 & 2.839 & 2.933 \\
5 & -13.653 & 1.563 & 1.234 & 1.128 & 1.135 & 1.168 & 1.190 \\
6 & 0.712 & 0.982 & 0.782 & 0.637 & 0.650 & 0.769 & 0.878 \\
7 & 3.372 & 0.983 & 0.792 & 0.694 & 0.727 & 0.824 & 0.910 \\
8 & -1.657 & 1.124 & 0.944 & 0.877 & 0.907 & 0.971 & 1.023 \\
9 & 6.283 & 0.976 & 0.832 & 0.801 & 0.841 & 0.897 & 0.943 \\
10 & 1.332 & 1.123 & 0.941 & 0.897 & 0.929 & 0.981 & 1.023 \\
11 & 1.225 & 1.112 & 0.945 & 0.886 & 0.909 & 0.963 & 1.011 \\
12 & 0.756 & 1.468 & 0.995 & 0.918 & 0.931 & 0.964 & 0.977 \\
13 & 1.229 & 1.197 & 1.008 & 0.944 & 0.970 & 1.028 & 1.022 \\
14 & 1.061 & 1.249 & 1.060 & 0.980 & 0.983 & 1.026 & 1.019 \\
\hline \hline
\end{tabular}

values and $\beta_{n}$ should have progressively increasing values till about 1.5 . If no care is taken in choosing the parameters, a large number of functions could be necessary for obtaining convergence. The results reported in this paper are obtained with the following parameters for the functions $f_{n}, n$ $=1, \ldots, 14: \quad\left\{\delta_{n}, \alpha_{n}, \beta_{n}, \gamma_{n}, \mu_{n}\right\} \equiv\{-0.5,-0.25,0.3,0.01$, $0.02\}, \quad\{-0.7,-0.25,0.5,0.04,0.02\}, \quad\{-0.7,-0.25,0.7$, $0.03,0.06\}, \quad\{-0.4,-0.1,0.6,0.2,0.2\}, \quad\{-0.2,0.1,0.8$, $0.2,0.2\}, \quad\{0.4,-0.2,0.6,0.3,0.3\}, \quad\{-0.2,-0.1,0.7,0.4,0.3\}$, $\{0.3,0.2,0.8,0.3,0.4\}, \quad\{0.2,0.2,1,0.4,0.4\}, \quad\{0.3,0.2,1.2$, $0.5,0.5\}, \quad\{-0.5,0.2,1.3,0.6,0.6\}, \quad\{-0.2,0.1,1.4,0.7,0.7\}$, $\{0.3,0,1.5,0.8,0.8\},\{-0.2,-0.1,1.6,0.9,0.9\}$. By employing a suitably chosen set of the parameters, we have kept the number of functions to a minimum.

In Table I, we show the convergence pattern of the present calculation with respect to the number of partial waves $L$ and basis functions $N$ used in the calculation. The convergence is satisfactory considering that we are dealing with a complicated five-body problem. However, as the present calculation does not produce a bound on the result, the convergence is not monotonic with increasing $N$. The final result of the present calculation is that for $N=14$ and $L=7: a=1.02$ a.u. Although it is difficult to provide a quantitative measure of convergence, from the fluctuation of this result for large $N$ and $L$ we believe the error in our result to be less than $10 \%$, so that the final Ps-He scattering length is taken as $a=(1.0$ $\pm 0.1)$ a.u. The results for large $N$ and $L$ reported in Table I all lie in this domain.

The maximum number of functions $(N=14)$ used in this calculation is also pretty small, compared to those used in different Kohn-type variational calculations for electronhydrogen $(N=56)$ [30], positron-hydrogen $(N \leqslant 286)$ [31], and positron-helium $(N \leqslant 502)$ [32] scattering. Because of the explicit appearance of the Green's function, the present basis-set approach is similar to the Schwinger variational method. Using the Schwinger method, convergent results for electron-hydrogen [33] and positron-hydrogen [34] scattering have been obtained with a relatively small basis set $(N$
$(\mathrm{N} \sim 10)$. These suggest a more rapid convergence in these problems with a Schwinger-type method.

\section{SUMMARY AND DISCUSSION}

To summarize, we have formulated a basis-set calculational scheme for $S$-wave Ps-He elastic scattering below the lowest inelastic threshold using a variational expression for the transition matrix. We illustrate the method numerically by calculating the scattering length in the electronic doublet state: $a=1.0 \pm 0.1$ a.u. This corresponds to a zero-energy cross section of $(4.0 \pm 0.8) \pi a_{0}^{2}$ in reasonable agreement with a model calculation by this author $\left(3.34 \pi a_{0}^{2}\right)$ [22] and the

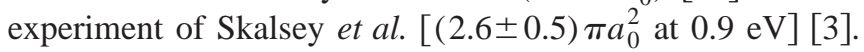
This calculation, as well as our previous studies of Ps-He scattering using a model exchange potential $[21,23]$, possibly consolidates the experimental result of Skalsey et al. However, these low-energy Ps-He elastic-scattering cross sections are in disagreement with other experiments by Nagashima

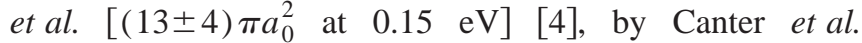
$\left(8.47 \pi a_{0}^{2}\right)$ [5], and by Coleman et al. $\left(9 \pi a_{0}^{2}\right)$ [6], as well as with conventional static-exchange, $R$-matrix, and closecoupling calculations of Refs. [8,11,12,15,17] $\left(\sim 14 \pi a_{0}^{2}\right)$ and a model potential calculation of Ref. [19] $\left(7.73 \pi a_{0}^{2}\right)$.

As the effective interaction for elastic scattering between $\mathrm{Ps}$ and $\mathrm{He}$ is repulsive in nature, a smaller scattering length as obtained in this paper and in Refs. [21,22] would imply a weaker effective Ps-He interaction. This would allow the Ps atom to come closer to $\mathrm{He}$ and would lead [25] to a large pickoff quenching rate and a large ${ }^{1} Z_{\text {eff }}(\sim 0.11)$ in agreement with experiment $[5,14]$. The conventional closecoupling [12], $R$-matrix [8] and static-exchange [11,15-17] models yielded a much too large scattering length corresponding to a stronger repulsion between Ps and He. Consequently, these models led to a much too small ${ }^{1} Z_{\text {eff }}$ $(\sim 0.04)[15-17,25]$ in disagreement with experiment $[5,14]$. This is addressed in detail in Ref. [25], where we established a correlation between the different scattering lengths and the corresponding ${ }^{1} Z_{\text {eff }}$. This correlation suggests that a small Ps-He scattering length as in this paper is consistent with the large experimental ${ }^{1} Z_{\text {eff }}$.

Although we have used a simple wave function for $\mathrm{He}$ in this complex five-body calculation, we do not believe that the use of a more refined He wave function would substantially change our findings and conclusions. However, independent calculations and accurate experiments at low energies are welcome for a satisfactory resolution of this controversy.

\section{ACKNOWLEDGMENTS}

The work is supported in part by the Conselho Nacional de Desenvolvimento-Científico e Tecnológico, Fundação de Amparo à Pesquisa do Estado de São Paulo, and Financiadora de Estudos e Projetos of Brazil. 
[1] A. J. Garner, A. Özen, and G. Laricchia, J. Phys. B 33, 1149 (2000)

[2] A. J. Garner, G. Laricchia, and A. Özen, J. Phys. B 29, 5961 (1996); N. Zafar, G. Laricchia, M. Charlton, and A. Garner, Phys. Rev. Lett. 76, 1595 (1996).

[3] M. Skalsey, J. J. Engbrecht, R. K. Bithell, R. S. Vallery, and D. W. Gidley, Phys. Rev. Lett. 80, 3727 (1998).

[4] Y. Nagashima, T. Hyodo, K. Fujiwara, and A. Ichimura, J. Phys. B 31, 329 (1998).

[5] K. F. Canter, J. D. McNutt, and L. O. Roellig, Phys. Rev. A 12, 375 (1975).

[6] P. G. Coleman, S. Rayner, F. M. Jacobsen, M. Charlton, and R. N. West, J. Phys. B 27, 981 (1994).

[7] A. J. Garner, A. Özen, and G. Laricchia, Nucl. Instrum. Methods Phys. Res. B 143, 155 (1998); P. K. Biswas, ibid. 171, 135 (2000)

[8] J. E. Blackwood, C. P. Campbell, M. T. McAlinden, and H. R. J. Walters, Phys. Rev. A 60, 4454 (1999).

[9] C. P. Campbell, M. T. McAlinden, F. G. R. S. MacDonald, and H. R. J. Walters, Phys. Rev. Lett. 80, 5097 (1998).

[10] P. K. Sinha, A. Basu, and A. S. Ghosh, J. Phys. B 33, 2579 (2000); P. K. Sinha, P. Chaudhury, and A. S. Ghosh, ibid. 30, 4643 (1997); H. Ray and A. S. Ghosh, ibid. 29, 5505 (1996).

[11] N. K. Sarkar and A. S. Ghosh, J. Phys. B 30, 4591 (1997).

[12] N. K. Sarkar, P. Chaudhury, and A. S. Ghosh, J. Phys. B 32, 1657 (1999).

[13] Z.-C. Yan and Y. K. Ho, Phys. Rev. A 60, 5098 (1999); A. M. Frolov and V. H. Smith, Jr., ibid. 55, 2662 (1997); N. Jiang and D. M. Schrader, J. Chem. Phys. 109, 9430 (1998).

[14] B. G. Duff and F. F. Heymann, Proc. R. Soc. London, Ser. A 270, 517 (1962); F. F. Heymann, P. E. Osmon, J. J. Veit, and W. F. Williams, Proc. Phys. Soc. London 78, 1038 (1961).
[15] M. I. Barker and B. H. Bransden, J. Phys. B 1, 1109 (1968).

[16] M. I. Barker and B. H. Bransden, J. Phys. B 2, 730 (1969).

[17] P. A. Fraser, J. Phys. B 1, 1006 (1968).

[18] P. A. Fraser and M. Kraidy, Proc. Phys. Soc. London 89, 553 (1966).

[19] R. J. Drachman and S. K. Houston, J. Phys. B 3, 1657 (1970).

[20] S. K. Adhikari and P. K. Biswas, Phys. Rev. A 59, 2058 (1999); S. K. Adhikari, ibid. 63, 054502 (2001).

[21] P. K. Biswas and S. K. Adhikari, Phys. Rev. A 59, 363 (1999).

[22] S. K. Adhikari, Phys. Rev. A 62, 062708 (2000).

[23] P. K. Biswas and S. K. Adhikari, Chem. Phys. Lett. 317, 129 (2000).

[24] P. K. Biswas and S. K. Adhikari, J. Phys. B 33, 1575 (2000).

[25] S. K. Adhikari, P. K. Biswas, and R. A. Sultanov, Phys. Rev. A 59, 4829 (1999).

[26] B. H. Bransden, Atomic Collision Theory (Benjamin, New York, 1970), p. 222.

[27] S. K. Adhikari, Phys. Rev. C 25, 118 (1982).

[28] S. K. Adhikari and I. H. Sloan, Phys. Rev. C 11, 1133 (1975); Nucl. Phys. A 241, 429 (1975); S. K. Adhikari, Phys. Rev. C 10, 1623 (1974); Variational Principles and the Numerical Solution of Scattering Problems (Wiley, New York, 1998).

[29] S. K. Adhikari and P. Mandal, J. Phys. B 34, L187 (2001); 33, L761 (2000).

[30] I. Shimamura, J. Phys. Soc. Jpn. 30, 1702 (1971).

[31] J. W. Humberston, P. VanReeth, M. S. T. Watts, and W. E. Meyerhof, J. Phys. B 30, 2477 (1997); E. A. G. Armour and J. W. Humberston, Phys. Rep 204, 1734 (1991).

[32] P. Van Reeth and J. W. Humberston, J. Phys. B 30, L95 (1997).

[33] K. Takatsuka and V. McKoy, Phys. Rev. A 30, 1734 (1984).

[34] S. Kar and P. Mandal, J. Phys. B 33, 2379 (2000); 32, 2297 (1999); Phys. Rev. A 59, 1913 (1999). 